09

\title{
Анализ вкладов различных факторов в магнитооптический сигнал трехмерных структур типа магнитофотонных кристаллов
}

\author{
(C) Н.Ю. Звездин, ${ }^{1}$ В.А. Папорков, ${ }^{1}$ А.В. Проказников, ${ }^{2, \uparrow}$ И.С. Царев ${ }^{1}$ \\ ${ }^{1}$ Ярославский государственный университет им. П.Г. Демидова, \\ 150000 Ярославль, Россия \\ ${ }^{2}$ Ярославский филиал Физико-технологического института РАН, \\ 150007 Ярославль, Россия \\ T e-mail: prokaznikov@mail.ru
}

(Поступило в Редакцию 13 декабря 2016 г.)

Впервые рассмотрено наложение эффектов разного уровня и порядков при формировании результирующего магнитооптического отклика от структур типа магнитофотонных кристаллов для различных длин волн падающего излучения в областях, далеких от плазмонных резонансов. Изучены вклады в магнитооптический отклик интерференционных и дифракционных явлений в максимумах различных порядков в трехмерных системах типа магнитофотонных кристаллов. Показано, что использование интегрального отклика для анализа магнитооптических эффектов приводит к исчезновению интерференционных явлений. Дифракционный максимум нулевого порядка адекватно отражает магнитную составляющую магнитооптического отклика. Проведены численные оценки наблюдаемых дифракционных и интерференционных эффектов.

DOI: $10.21883 /$ JTF.2018.06.46022.2133

\section{Введение}

В настоящее время одним из распространенных методов исследования магнитных и магнитооптических (MO) свойств структур, особенно в нанометровом диапазоне размеров, являются магнитооптические измерения различных характеристик, прежде всего магнитооптического экваториального эффекта Керра (МОЭЭК). Особый интерес представляют структуры, обладающие свойством пространственной периодичности. Подобные магнитные структуры носят название магнито-фотонных кристаллов и обладают рядом уникальных свойств. При определенных условиях эксперимента в них наблюдается резкий рост отклика системы на внешнее воздействие электромагнитным излучением $[1,2]$.

Особое внимание уделяется магнитным и магнитооптическим свойствам периодических структур микронных и субмикронных размеров различной формы, особенно точкам и полоскам. Изучение подобных массивов субмикронных элементов является основой для разработки наноструктурированной записывающей информацию среды и магнитной памяти на ее основе, в то время как взаимодействие между элементами определяет пределы интеграции магнитной памяти и записывающих сред. Эффекты взаимодействия между элементами в массивах гораздо меньше исследованы по сравнению с размерными эффектами. Магнитооптическая техника и, в частности, дифракционный магнитооптический эффект Керра (ДМОЭК) являются наиболее подходящими для исследования подобных явлений, поскольку они проявляют высокую чувствительность к фиксации малейших изменений намагниченности тонких пленок и очень малых элементов [3]. ДМОЭК предоставляет ценную информацию о распределении намагниченности и ее анизотропии вследствие необычайной чувствительности метода к магнитным неоднородностям [3].

Как правило, ДМОЭК наблюдался на периодических плоских двумерных структурах. В работах [4-9] исследовались металлические магнитные пленки, в которых имелись периодически расположенные отверстия круглой [6], квадратной [8] и эллиптической [9] форм. При разной ориентации пленки относительно плоскости падения света измерялись магнитооптические петли гистерезиса (МОПГ) в различных дифракционных порядках. Обнаружено, что петля, измеренная на нулевом дифракционном максимуме, не обладает какими-либо особенностями, тогда как на максимумах высших порядков они имеют место [8]. В работе [5] исследовались образцы, представляющие собой микрорешетку в виде регулярно расположенных островков кобальта квадратной формы размером $2 \times 2 \mu \mathrm{m}^{2}$ и расстоянием между ними от 0.2 до $2 \mu \mathrm{m}$. У всех исследованных пленок петли гистерезиса, измеренные в первом и высших дифракционных порядках, обладали особенностями, подобными представленным в работе [8].

В развитых к настоящему времени технологиях изготовления магнитофотонных структур высота единичной элементарной ячейки много меньше длины волны падающего электромагнитного излучения. Тенденции развития современной электроники, однако, диктуют необходимость использования трехмерных объектов с различными масштабами размеров по сравнению с длиной волны падающего излучения по всем направлениям, в том числе и по высоте сформированных структур [10]. Разнообразие конфигураций современных трехмерных структур охватывает области от массивов вертикальных колонн, в том числе и с изменяющимся поперечным сечением, до трехмерных голографических фотонных 
$a$

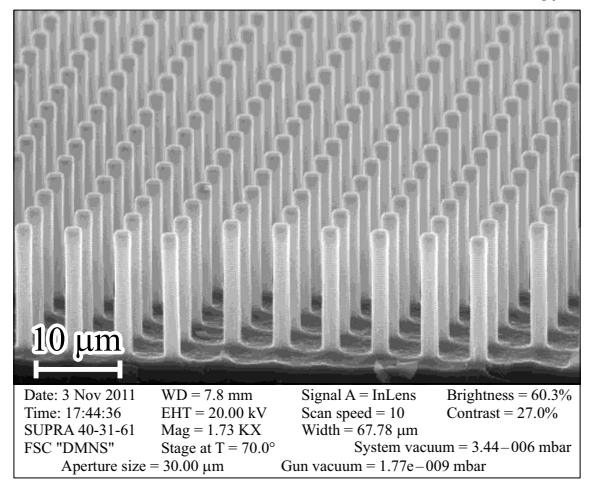

$b$

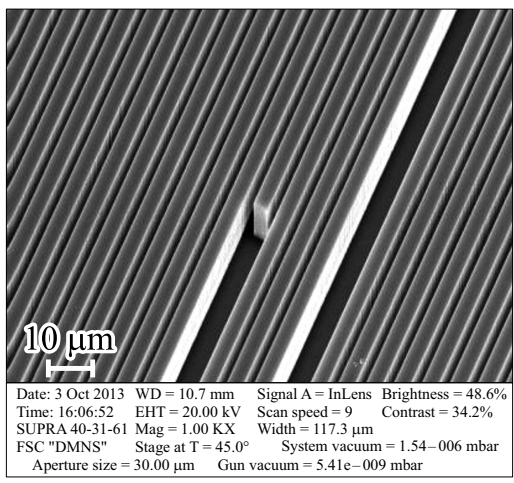

C

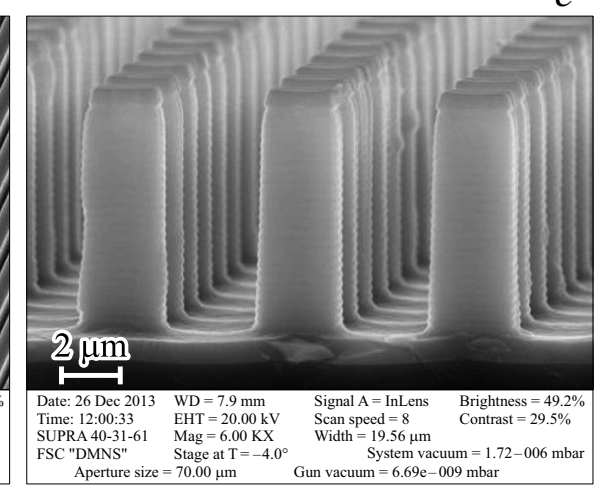

Рис. 1. Кремниевые структуры: $a-$ в форме вертикальных „круглых“ колонн с напылением слоя кобальта толщиной $6 \mathrm{nm;} b-$ в форме полосок с вертикальными стенками с напылением слоя кобальта толщиной $6 \mathrm{~nm} ; c-$ в виде прямоугольных колонн с напылением слоев золота толщиной $10 \mathrm{~nm}$ (снизу) и кобальта толщиной $6 \mathrm{~nm}$ (сверху) на кремний.

кристаллов, работающих в оптическом окне связи [10]. Все это диктует необходимость разработки адекватных методов измерения и интерпретации отклика подобных сложных трехмерных систем на внешние электромагнитные воздействия, в том числе при наличии дополнительных внешних полей, прежде всего, магнитного, в методиках регистрации магнитооптического эффекта Керpa [11].

\section{Технология изготовления структур и методика проведения эксперимента}

Измерения производились на структурах двух типов. При создании образца первого типа на базе стандартных кремниевых пластин КЭФ-4.5 с ориентацией (100) формировались вертикальные „колонны“ плазмохимическим травлением посредством стандартного Bosch-процесса через хромовую $(\mathrm{Cr})$ маску толщиной $30 \mathrm{~nm} \mathrm{c} \mathrm{ис-}$ пользованием взрывной литографии (lift-off lithography). Результат описанной процедуры при различных параметрах травления представлен на рис. 1. Отметим, что Bosch-процесс является циклическим двустадийным процессом травления, который включает чередующиеся процессы, в частности, травления в составе $\mathrm{SF}_{6}(2-5 \mathrm{~s})$ и пассивации в $\mathrm{C}_{4} \mathrm{~F}_{8}(2-5 \mathrm{~s})$. Чередование этих процессов обусловливает характерную структуру боковых стенок, сформированных при анизотропном травлении $[12,13]$. Отметим, что результат снятия верхней маски из хрома на структуре на рис. 1, $a$ выразился как в уменьшении поперечных размеров, так и в небольшом травлении острых углов у столбчатых структур, так что конечная форма сечения колонн отличалась от прямоугольной, и была близка к круглой. Структуры второго типа в форме полосок (рис. $1, b)$ формировались похожим способом, но использовалась маска из полимерного покрытия, поэтому изменений структуры на этапе снятия маски не наблюдалось. Структуры третьего типа в виде колонн с прямоугольным сечением содержали (рис. 1,c) слои зо- лота толщиной $10 \mathrm{~nm}$ (снизу) и кобальта толщиной $6 \mathrm{~nm}$ (сверху) на поверхности кремния.

На следующем этапе для структур обоих видов магнетронным напылением наносился слой кобальта (Co) толщиной $6 \mathrm{~nm}$, включая нижнее пространство между структурами (дно массива структур) и их стенки. Один из образцов со структурами в виде продольных полосок после напыления слоя Со отжигался в вакууме при температуре $400^{\circ} \mathrm{C}$ в течение $60 \mathrm{~min}$, в то время как другой образец не подвергался отжигу.

В настоящей работе исследовались МОПГ и угловые зависимости амплитудных значений МОЭЭК и коэффициентов отражения в дифракционных максимумах различных порядков, измеренные при двух ортогональных ориентациях образца относительно направления внешнего магнитного поля. Исследования проводились при комнатной температуре согласно методике, детально изложенной в работе [14]. Образец помещался между полюсами электромагнита, создающего магнитное поле частотой $30 \mathrm{~Hz}$ с амплитудой до 400 Ое, достаточной для магнитного насыщения образца [14]. Поляризованный в плоскости падения ( $p$-волна) лазерный пучок света диаметром $1 \mathrm{~mm}$ падал на поверхность пленки под разными углами и при разной ориентации образцов относительно плоскости падения и магнитного поля в стандартной конфигурации для измерений МОЭЭК (направление магнитного поля перпендикулярно плоскости падения). Измерялась величина

$$
\delta=\Delta I / I(0)
$$

где $\Delta I=I(H)-I(0)$. Здесь $I(H)-$ интенсивность света, отраженного от намагниченной поверхности; а $I(0)-$ интенсивность света, отраженного от ненамагниченной поверхности; $H$ - напряженность магнитного поля. Значение $\Delta I$ пропорционально переменной составляющей тока фотоприемника, $\mathrm{I}(0)$ пропорционально постоянной составляющей тока. Коэффициент отражения определялся как отношение постоянной составляющей интен- 


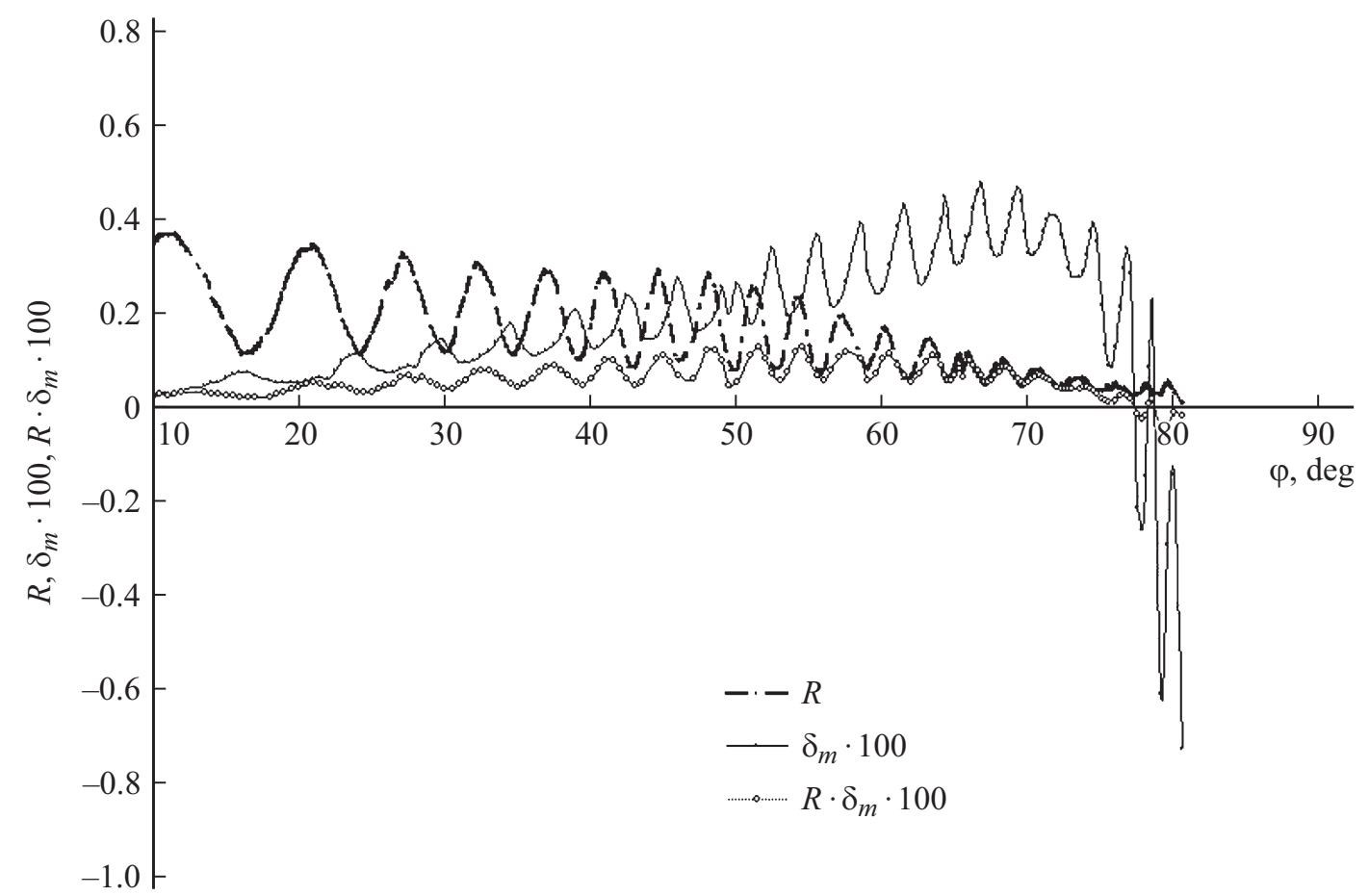

Рис. 2. Зависимость $R(\varphi), \delta_{m}(\varphi), R(\varphi) \delta_{m}(\varphi)$ на структуре типа вертикальных полосок. Плоскость падения света совпадает с направлением полосок; $\lambda=633 \mathrm{~nm}$.
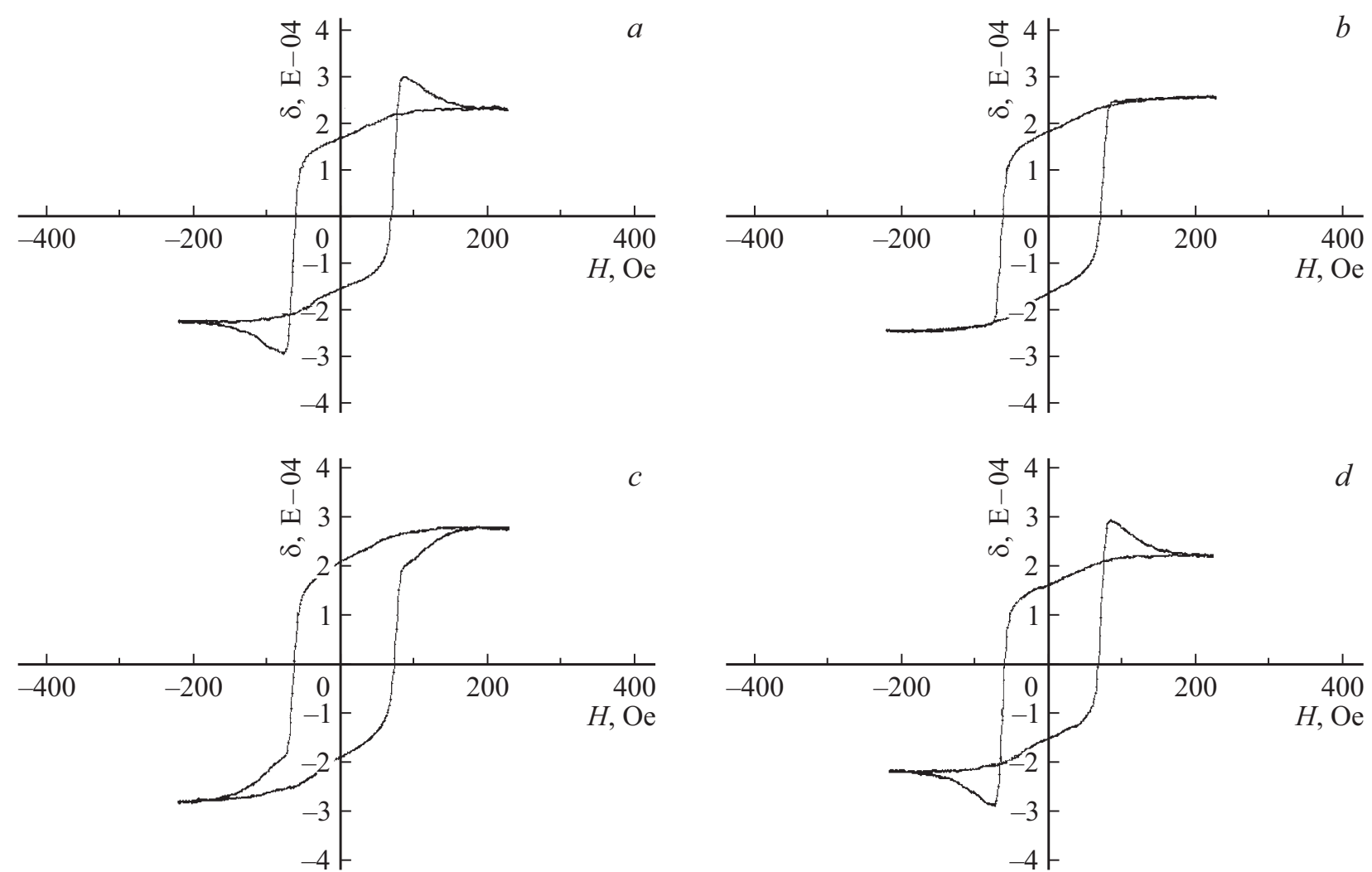

Рис. 3. Периодическое изменение формы петли гистерезиса от $a$ к $d$ в зависимости от угла падения света на образец для структур типа вертикальных колонн (первого типа). Направление падающего луча - вдоль рядов колонн. Угол падения: $a-57.5^{\circ}, b-$ $58.0^{\circ}, c-58.5^{\circ}, d-59.0^{\circ}, \lambda=633 \mathrm{~nm}$. 
сивности отраженной волны $I(0)$ к интенсивности $I_{\text {in }}$ падающей волны: $R=I(0) / I_{i n}$. Зависимость величины $\delta$ от напряженности магнитного поля $\delta(H)$ представляла собой МОПГ. Для построения угловых зависимостей величины МОЭЭК использовалось амплитудное значение $\delta_{m}=\delta\left(H_{\max }\right)$, где $H_{\max }-$ амплитудное значение напряженности магнитного поля.

Для пространственного разрешения дифракционных максимумов использовались пластинки-экраны с отверстиями. Пластинка располагалась на пути отраженного луча в непосредственной близости от исследуемой пленки. Поскольку без применения дополнительных технических средств во входной зрачок фотоприемника попадал дифракционный максимум только нулевого порядка, для измерения максимумов высших порядков дополнительно использовался длиннофокусный объектив $(f=28 \mathrm{~mm})$ с апертурным углом $22^{\circ}$. Этот же объектив применялся для формирования изображения освещаемого участка поверхности пленки в плоскости фотоприемника. В последнем случае измерялся интегральный (суммарный) магнитооптический эффект. При измерении нулевого дифракционного максимума угол падения света изменялся от $7^{\circ}-10^{\circ}$ до $85^{\circ}-87^{\circ}$ с шагом $0.5^{\circ}$, а в области смены знака МОЭЭК $-0.25^{\circ}$. Использование объектива при измерении интегрального магнитооптического отклика и дифракционных максимумов высших порядков не позволяло провести измерения в таком широком интервале углов. В этом случае угол изменялся от $25^{\circ}$ до $50^{\circ}$ с шагом $1^{\circ}$. Источниками света служили лазеры с длинами волн $\lambda=0.633$ и $0.405 \mu \mathrm{m}$. Максимальный дифракционный порядок, наблюдаемый при использовании этих лазеров, составлял 1 и 2 соответственно.

\section{Основные экспериментальные результаты и их обработка при измерении дифракционного максимума нулевого порядка}

На рис. 2 приведены результаты измерений угловых зависимостей коэффициента отражения $R(\varphi)$ и величины МОЭЭК $\delta_{m}(\varphi)$ на структуре второго (полоски) типа. На графиках четко прослеживаются осцилляции этих зависимостей. Период осцилляций уменьшается с увеличением угла падения. При углах $75^{\circ}-80^{\circ}$ происходила инверсия МОПГ. На угловой зависимости $\delta_{m}(\varphi)$ это соответствовало смене знака величины МОЭЭК (рис. 2). Подобные зависимости с отмеченными выше характерными закономерностями наблюдались и на структуре первого типа (вертикальные колонны). Типичные МОПГ, измеренные на этой структуре, при нескольких углах падения представлены на рис. 3 .

Из рис. 2 видно, что максимумы коэффициента отражения $\max R(\varphi)$ совпадают с минимумами магнитооптического эффекта $\min \delta_{m}(\varphi)$. Максимумам $R(\varphi)$ соответствовали МОПГ, показанные на рис. 3, $c$; мимумам $R(\varphi)$ - МОПГ, показанные на рис. $3, a, d$.
В формуле (1), определяющей величину МОЭЭК, числитель дроби $\Delta I=I(H)-I(0)$ зависит от магнитного поля и в этом смысле является магнитной составляющей эффекта. Величина $I(0)$, пропорциональная коэффициенту отражения, является его оптической составляющей. Поэтому если величину эффекта $\delta_{m}(\varphi)$ умножить на $R(\varphi) \sim I(0)$, то можно выделить магнитную составляющую МОЭЭК $\Delta I \sim R(\varphi) \delta_{m}(\varphi)$ и построить ее угловую зависимость. Эта зависимость для структуры второго типа показана на рис. 2. У всех структур $\max R(\varphi)$ совпадают с $\max (R(\varphi) \delta m(\varphi))$. Осцилляции $\delta_{m}(\varphi)$ свидетельствуют о различии вкладов в величину МОЭЭК числителя, определяемого магнитными свойствами системы, и знаменателя, пропорционального коэффициенту отражения. Графики, представленные на рис. 2, свидетельствуют о том, что разные эффекты дают свой вклад в общий конечный результат МОЭЭК. Характерным свойством графиков, представленных на рис. 2, является согласованность их осцилляций.

Наиболее ярко осциллирующие зависимости величины МОЭЭК проявляются при дополнительной обработке экспериментальных данных, принцип которой схематично представлен на рис. 4. Как показали проведенные детальные исследования различных вариантов представления результата, одной из информативных характеристик, которая в большей степени отражает периодическую зависимость магнитооптических характеристик от угла падения света, является разность $D=(\mathrm{Gr}-\mathrm{Rem})$. Величины Gr и Rem схематично представлены на рис. 4.

Петли гистерезиса, представленные на рис. 4, соответствуют структурам типа вертикальных колонн (первого типа) и неотожженным структурам второго типа (полоски) (рис. 1) в конфигурации, когда магнитное поле направлено перпендикулярно узкой грани структуры, а плоскость луча падающего света направлена вдоль полосок. Подобные петли гистерезиса, форма которых зависит от угла падения, наблюдаются лишь при определенных ориентациях плоскости падения света относительно рядов колонн или полосок.

Отмеченная закономерность может быть представлена в виде наложения двух петель с различной формой и динамикой изменения в зависимости от угла падения света, что схематично показано на рис. 4. Результирующие петли гистерезиса в этом случае определяются следующим образом: при $\max R(\varphi)-$ это $\delta_{1}=\delta_{a}+\delta_{b}$, при $\min R(\varphi)-$ это $\delta_{2}=\delta_{a}-\delta_{b}$, где $\delta_{1}-$ МОПГ первого типа (рис. $3, c ; 4, a), \delta_{2}-$ МОПГ второго типа (рис. $3, a, d ; 4, b) ; \delta_{a}$ и $\delta_{b}-$ петли гистерезиса двух участков „, $a^{\text {“ }}$ и „, $b^{\text {c }}$ пленки, на которых формируется отраженный сигнал. На рис. 4, $a$ петли $\delta_{a}, \delta_{b}$ неинвертированы, тогда как на рис. $4, b$ петля $\delta_{a}$ неинвертирована, а $\delta_{b}$ инвертирована.

Для проверки этой гипотезы брались петли гистерезиса, измеренные на структуре с полосками при двух близких углах падения, для которых наблюдались петли первого $\left(\delta_{1}\right)$ и второго $\left(\delta_{2}\right)$ типов. Эти петли схематично показаны на рис. 4. На их основе строились петли 

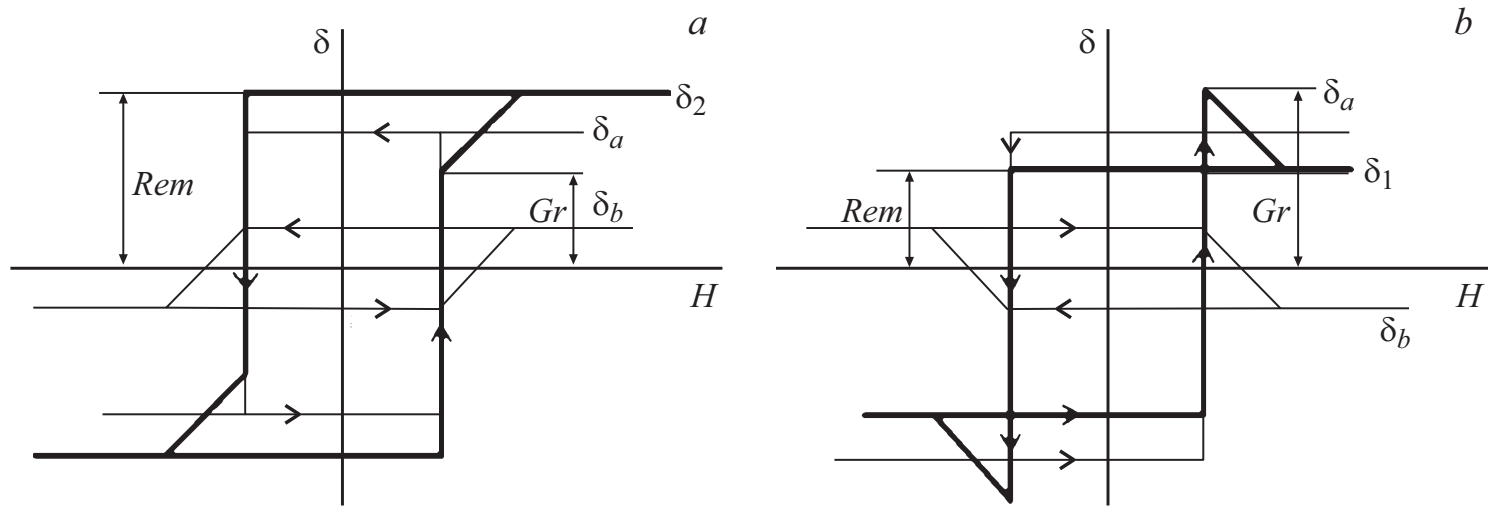

Рис. 4. Схематичное представление петли гистерезиса с разложением на составные элементы (тонкие линии), которые в суперпозиции дают результирующую зависимость, наблюдаемую на эксперименте (жирные линии).
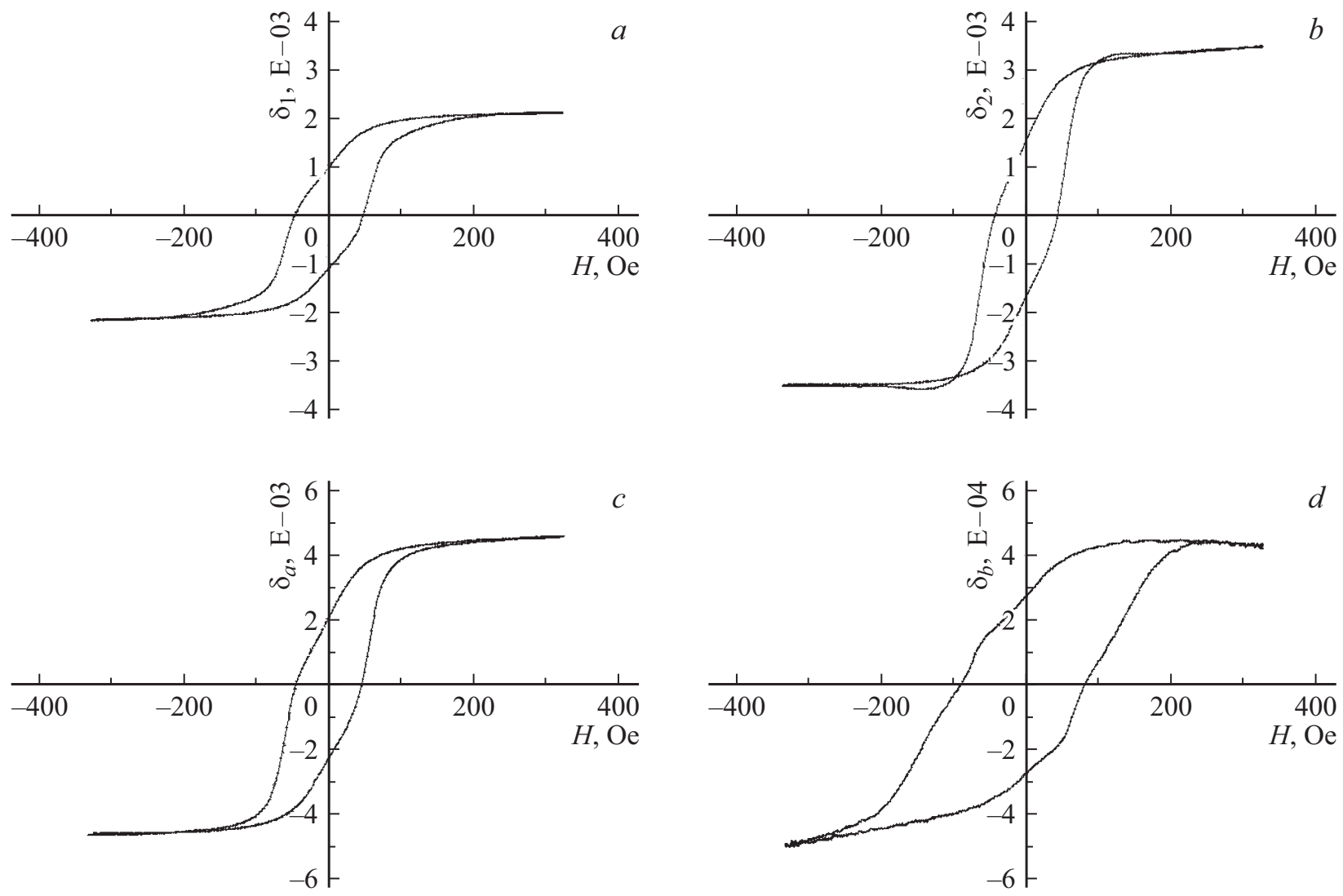

Рис. 5. Петли гистерезиса, измеренные на структуре второго типа (полоски) при углах падения: $a-52.5^{\circ}\left(\delta_{1}\right)$ и $b-54.0^{\circ}\left(\delta_{2}\right)$,

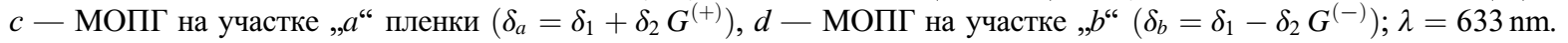

$\delta_{a}=\delta_{1}+\delta_{2} G^{(+)}, \delta_{b}=\delta_{1}-\delta_{2} G^{(-)}$, где $G^{(+)}$- эмпирические коэффициенты $(\sim 1)$ для неинвертированной $(+)$ и инвертированной (-) МОПГ.

Результат такого построения для структуры второго типа (полоски) представлен на рис. 5. На рис. 5, $a$ приведена петля первого типа $\left(\delta_{1}\right)$, петля второго типа $\left(\delta_{2}\right)$ показана на рис. $5, b$. Петля $\delta_{a}=\delta_{1}+\delta_{2} G^{(+)}$изображена на рис. $5, c$, на рис. $5, d-$ петля $\delta_{b}=\delta_{1}-\delta_{2} G^{(+)}$, (здесь $G^{(-)}=0.48, G^{(+)}=0.67$ ). Из рис. $5, c, d$ видно значительное отличие магнитных свойств участков, фор- мирующих магнитооптический сигнал. Такими участками являются верхняя и нижняя (дно) поверхности периодических структур (рис. 1).

В нашем случае на рис. 5 петля $\delta_{a}-$ МОПГ на верхней поверхности полосок, а $\delta_{b}-$ на нижней. Величины МОЭЭК этих участков отличаются на порядок. Причиной последнего может быть разная толщина слоя Со на них, связанная с особенностями напыления Со на такую структуру. В работах $[15,16]$ показано, что коэрцитивная сила однородной тонкой пленки меньше коэрцитивной 
силы островковой пленки при плотном расположении ферромагнитных островков. В нашем случае, кроме этого, величина эффекта от дна структуры существенно меньше, чем от поверхности, поскольку в более тонкой пленке в знаменателе формулы (1) значительную роль начинает играть сигнал, отраженный от немагнитной подложки. На магнитооптических петлях гистерезиса, измеренных на аналогичном образце, подвергнутом отжигу, форма петли не зависела от угла падения, что свидетельствовало об одинаковости магнитных свойств участков пленки, формирующих отклик. Это обусловлено растеканием островков в результате отжига с образованием однородной пленки. При этом осцилляции $R(\varphi)$ и $\delta_{m}(\varphi)$ не изменились.

Измерения, выполненные на всех структурах при ориентации плоскости падения света под углом $45^{\circ}$ относительно направления рядов колонн или полосок, показали отсутствие отмеченных выше осцилляций при углах падения больше $15^{\circ}$.

\section{Обсуждение эффректов интерференции для нулевого порядка дифракционного максимума}

В направлении вдоль вертикальных стенок структур имеет место иная ситуация по сравнению с отражением света от верхней и нижней горизонтальных поверхностей структуры. В узких каналах между структурами возможно многократное рассеяние света по типу интерференции Фабри-Перо. Может также иметь место и интерференция лучей, отраженных от верхней и нижней (дна) плоскостей структуры. Проведем анализ вклада в наблюдаемые закономерности, который вносит интерференция света от верхних и нижних граней в виде плоских участков сложной трехмерной металлизированной магнитным материалом структуры. Оценим угловые расстояния между максимумами (минимумами) в случае интерференции двух лучей, один из которых отражается от верхней части структур, изображенных на рис. 1, а другой - от дна структуры.

Поскольку измерялась интенсивность дифракционного максимума нулевого порядка, а именно в нем в основном сосредоточена интенсивность отраженной волны, угол наблюдения (измерения) равнялся углу падения. В этом случае можно воспользоваться стандартным подходом, изложенным, например, в работе [17]:

$$
2 h \cos \varphi_{m}= \pm \lambda m^{*},
$$

где $h$ - высота структуры, $m^{*}$ - порядок интерференционного максимума, $m^{*}=0,1,2,3 \ldots, \varphi_{m}-$ соответствующий ему угол падения.

При $m^{*}=0$ угол $\varphi=90^{\circ}$. Поскольку величина $\varphi$ не превышала $85^{\circ}-87^{\circ}$, определить мимальное значение $m^{*}$ затруднительно. В этом случае разумно отсчитывать порядок максимумов по-другому - со сто- роны малых углов, т.е. сделать замену $m=M-m^{*}$, где $M$ - максимальное значение $m^{*}, m-$ порядковый номер экстремума. В зависимости от высоты $h$ и длины волны $\lambda$ значение $M=\operatorname{int}(2 h / \lambda)$ находилось в интервале $10<M<20$. График зависимости $\cos \varphi_{m}$ от $m$ представляет собой убывающую линейную функцию

$$
\cos \varphi_{m}=(M-m) \lambda /(2 h)
$$

Аппроксимировав эту зависимость линейной функцией по тангенсу угла ее наклона, равному $\lambda /(2 h)$, можно найти $h$. На рис. 6 представлены зависимости $\cos \varphi_{m}$ от $m$ для структуры второго типа (полоски) для $R(\varphi)$ и $\delta_{m}(\varphi)$. Из графиков было определено значение $h_{1}^{\exp 1}=7.0 \mu \mathrm{m}$ для осцилляций $R(\varphi)$ и $h_{2}^{\exp }=7.4 \mu \mathrm{m}$ для осцилляций $\delta_{m}(\varphi)$. Из рис. 2 можно определить высоту полосок $h^{\text {micr }}$, наблюдаемых с помощью электронного микроскопа. Поскольку снимок делался под углом $28^{\circ}$ относительно плоскости пленки, полученный результат $h^{\text {micr }}=6.4 \mu \mathrm{m}$ следует разделить на $\cos \left(28^{\circ}\right)$. В результате $h=7.2 \mu \mathrm{m}$, что хорошо согласуется с измерениями для $R(\varphi)$ и $\delta_{m}(\varphi)$.

При малых углах падения из (2) следует

$$
2 h \cos \varphi \approx 2 h\left(1-\frac{\varphi^{2}}{2}\right)=\lambda m^{*},
$$

$m^{*}=0,1,2,3 \ldots$.

После преобразований получим

$$
2 h-h \varphi^{2}=\lambda m^{*} .
$$

Поскольку $2 h=M \lambda$, получим

$$
\lambda M-\lambda m^{*}=h \varphi^{2}=\lambda m, m=0,1,2,3 \ldots .
$$

Откуда следует выражение для угла, определяющего экстремум:

$$
\varphi_{m}=\sqrt{\frac{\lambda m}{h}},
$$

и периода осцилляций $R(\varphi)$ :

$$
\Delta \varphi_{m}=\varphi_{m+1}-\varphi_{m}=\sqrt{\frac{(m+1) \lambda}{h}}-\sqrt{\frac{m \lambda}{h}},
$$

которое при $m \gg 1$ приводит к выражению

$$
\Delta \varphi_{m} \approx \frac{1}{2} \sqrt{\frac{\lambda}{h m}}
$$

Отметим, что если выразить $\cos \varphi_{m}$ непосредственно из выражения (2), то получим $\cos \varphi_{m}=\frac{\lambda m^{*}}{2 h}$, кратно половине длины волны. Определенный вклад вносит периодическое прохождение и блокировка пучков света в каналах структур. Положим $a+b=d$, где $a-$ характерный размер торца структуры, $b-$ расстояние между отдельными структурами, $d-$ период структур. 

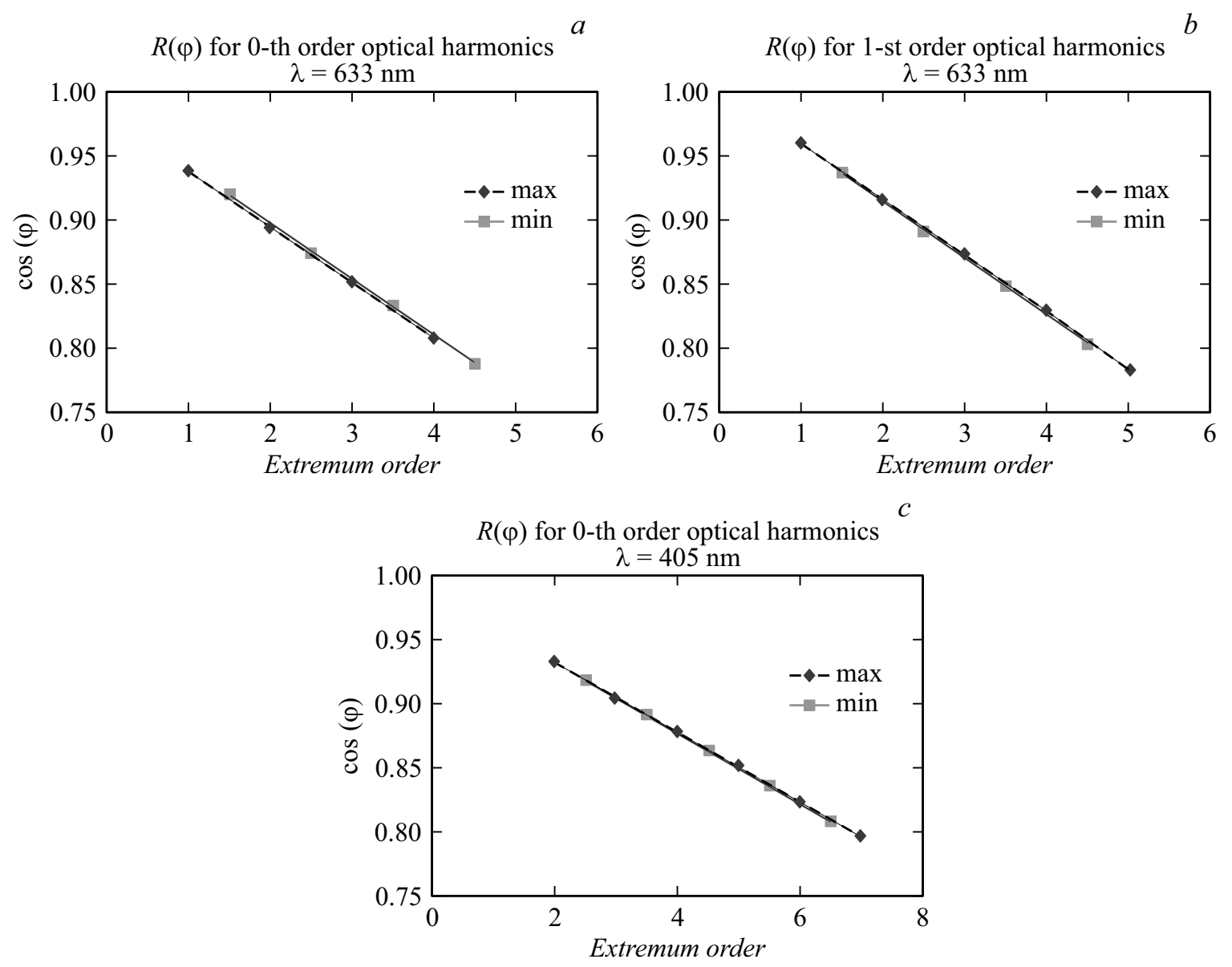

Рис. 6. Зависимость косинуса угла падения, соответствующего экстремумам угловой зависимости $R(\varphi)$ нулевого и первого дифракционных максимумов от порядкового номера экстремума $m$, для $\lambda=633 \mathrm{~nm}(a, b)$ и $\lambda=405 \mathrm{~nm}(c)$ на структуре второго типа (полоски).

Меньшее отражение при интерференции в канале вертикальной структуры происходит при следующих углах:

$$
\operatorname{tg} \varphi_{m}=\left(\frac{2 m+1}{2}\right) \frac{b}{h}, m=0,1,2,3 \ldots
$$

Большее отражение при интерференции в канале вертикальной структуры происходит при следующих углах:

$$
\operatorname{tg} \varphi_{m}=\frac{b m}{h}, m=0,1,2,3 \ldots
$$

При подстановке характерных размеров структур в микрометровом диапазоне, изображенных на рис. 1, было получено хорошее согласие в численных оценках наблюдаемых эффектов, прежде всего периодических изменений и характерного уменьшения периода с увеличением угла падения светового луча, отсчитываемого от нормали к плоскости падения (рис. 6).

Воспользовавшись методом, предложенным в работе [11], оценим высоту $h$ микрополосок, на которых происходит интерференция отраженных от поверхности волн. Для этого построим зависимость $\cos \varphi_{m}$ от $m$, соответствующую экстремумам угловой зависимости $R(\varphi)$ нулевого и первого дифракционных максимумов от порядкового номера $m$ экстремума. Эти графики представлены на рис. 6 для обеих длин волн.

Тангенс угла наклона такой зависимости $\operatorname{tg} \alpha=$ $=\alpha /(2 h)$ [18]. Для $\lambda=633 \mathrm{~nm}$ получилось следующее: $h=7.25 \mu \mathrm{m}(\max ), h=7.15 \mu \mathrm{m}(\min )$ для нулевого дифракционного максимума и $h=7.08 \mu \mathrm{m}$ $(\max ), h=7.15 \mu \mathrm{m}(\mathrm{min})$ для первого дифракционного максимума. Для $\lambda=405 \mathrm{~nm} h=7.38 \mu \mathrm{m}$ (max), $h=7.40 \mu \mathrm{m}(\mathrm{min})$. Представленные результаты хорошо согласуются со значением $h=7.2 \mu \mathrm{m}$, полученным с помощью электронного микроскопа, что также подтверждает интерференционную природу осцилляций рассмотренных зависимостей.

Об интерференционном характере отмеченных выше явлений свидетельствует отсутствие осцилляций при ориентации плоскости падения относительно рядов периодических структур в $45^{\circ}$ для углов падения больше $15^{\circ}$. При больших углах падения в такой ориентации возникает интенсивное рассеяние света на вертикальных элементах структуры. Для оценки этого угла можно воспользоваться формулой (10) при $m=1$. Подстановка в нее величин $b$ и $h$ из рис. 1 дает величину порядка $15^{\circ}$. 

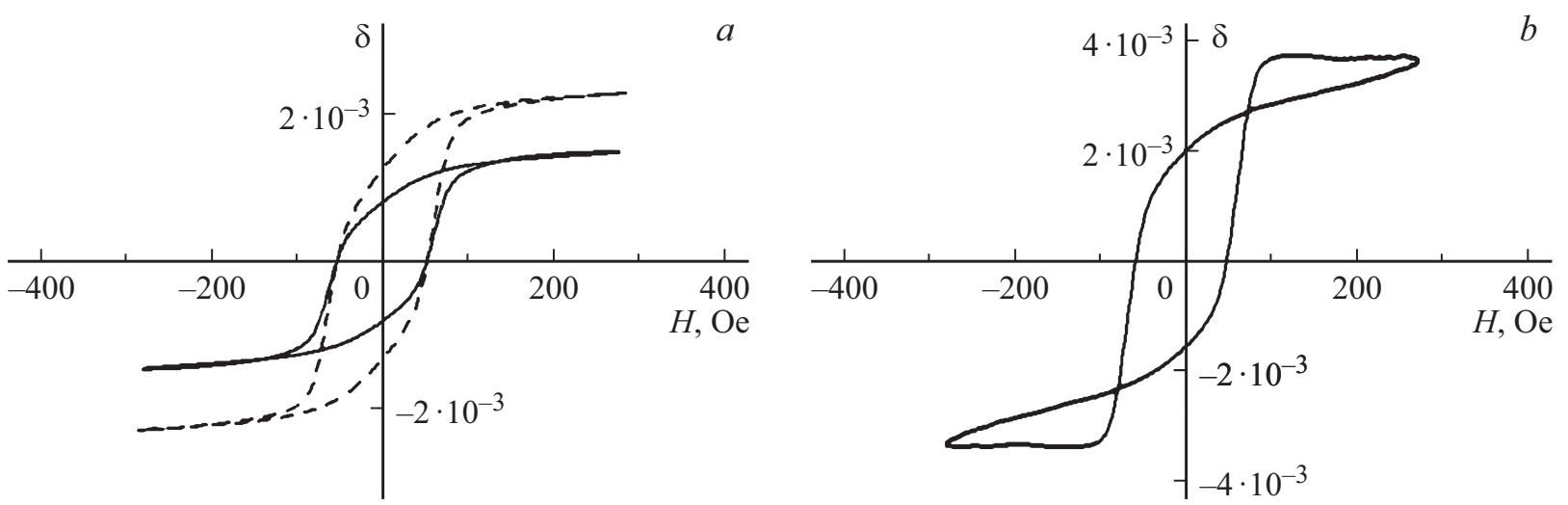

Рис. 7. $a-$ МОПГ, измеренные при $38^{\circ}$ на нулевом дифракционном максимуме (штрих) и с использованием объектива (сплошная линия); $b$ - МОПГ, измеренная при $36^{\circ}$ на первом дифракционном максимуме; $\lambda=633 \mathrm{~nm}$.

\section{Результаты измерений для дифракционных максимумов нулевого, высших порядков и их суперпозиции}

Как отмечалось выше, при измерении нулевого дифракционного максимума угол падения света изменялся от $7^{\circ}$ до $87^{\circ}$, а при измерении интегрального магнитооптического отклика и дифракционных максимумов высших порядков - от $25^{\circ}$ до $50^{\circ}$. Исследовался заданный угловой интервал, и по приведенным выше формулам (3), (7), (9) вычислялось количество экстремумов в заданном интервале углов падения излучения на образец. С ростом угла падения расстояние между экстремумами уменьшается, что находится в хорошем согласии с формулой (9). Эти факты приводят к заключению, что все отмеченные эффекты необходимо учитывать при анализе результатов магнитооптических измерений подобных структур. Таким образом, результирующий отклик системы представляет собой совокупный результат сложного наложения интерференционных и дифракционных явлений, а также содержит информацию о свойствах индивидуального рассеивателя. В случае, когда магнитные свойства поверхностей, формирующих магнитооптический отклик, различаются, форма магнитооптической петли гистерезиса зависит от угла падения света и определяется интерференцией волн, отраженных от горизонтальных и вертикальных плоскостей ступенчатой структуры. При этом характер осцилляций угловых зависимостей величины МОЭЭК и коэффициента отражения определяется ориентацией плоскости падения света относительно рядов колонн или полос микроструктуры, размерами структурных элементов и не зависит от магнитных свойств поверхностей. Для детальной интерпретации наблюдаемых эффектов необходимо учесть, что для отожженных образцов со структурой в виде полос, изображенных на рис. 1, $b$, имеет место характерная зависимость без наличия структуры типа „гребня“ на петлях гистерезиса. Таким образом, магнитные свойства отожженной структуры становятся более однородными по объему, тогда как у неотоженных структур имеет место различие между магнитными свойствами пленки, напыленной на верхние грани структуры, и пленки на боковых стенках и дне соответствующих структур. Этот факт оказывает существенное влияние на формирование результирующего отклика в исследуемых, вообще говоря, трехмерных структурах (рис. 1). Результирующий отклик системы формируется наложением эффектов от верхних граней, нижних граней и боковых стенок. Если магнитные свойства этих слоев отличаются друг от друга, то формируются петли, схема которых изображена на рис. 3, которая состоит из наложения двух различных петель согласно схеме, изображенной на рис. 3 .

На рис. 7 представлены типичные МОПГ, измеренные на нулевой и первой оптических гармониках, а также с использованием объектива для $\lambda=633 \mathrm{~nm}$. Из рис. 7 видно, что первые две петли подобны друг другу, а петля, измеренная на первом дифракционном максимуме (сравни с результатами работы [8]), значительно от них отличается. Коэрцитивные силы у всех МОПГ одинаковы.

Измерения делались в широком интервале углов падения света. На рис. 7 показаны МОПГ, измеренные на нулевом дифракционном максимуме и с использованием объектива в интервале углов падения $38.0^{\circ}-40.5^{\circ}$ для $\lambda=633 \mathrm{~nm}$. Из рисунка видно, что уже в достаточно узком интервале изменения угла падения значение $\delta_{m}$, измеренное на нулевом дифракционном максимуме, существенно меняется, тогда как $\delta_{m}$, измеренное при использовании объектива, практически остается постоянным.

На рис. 8 показаны угловая зависимость $\delta_{m}(\varphi)$ для нулевого дифракционного максимума, угловые зависимости $R_{0}(\varphi)$ для нулевого и $R_{1}(\varphi)$ для первого дифракционных максимумов, а также $R_{\text {sum }}(\varphi)-$ суммарный оптический отклик, измеренный с использованием объектива, для $\lambda=633 \mathrm{~nm}$. На всех графиках четко прослеживаются осцилляции этих зависимостей, 

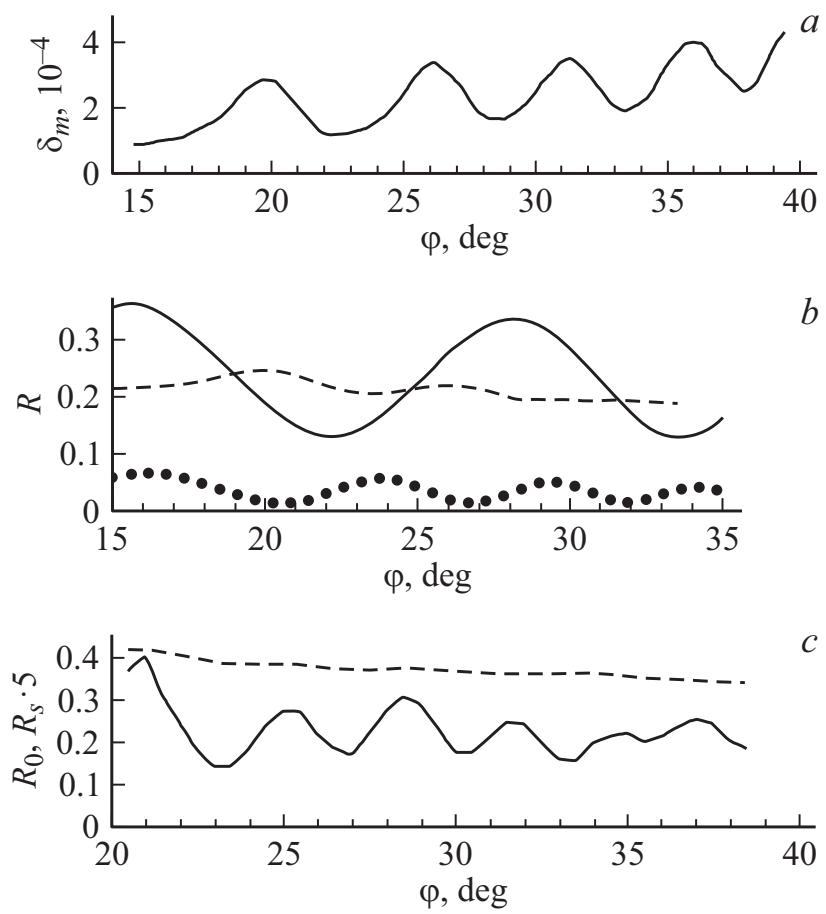

Рис. 8. График зависимости: $a-\delta_{m}(\varphi)$ при измерениях на нулевом дифракционном максимуме, $b-R_{0}(\varphi)$ (сплошная линия), $R_{1}(\varphi)$ (точки), $R_{\text {sum }}(\varphi)$ (штрих) для $\lambda=633 \mathrm{~nm}, c-$ график зависимости $R(\varphi)$ для нулевого дифракционного максимума (сплошная линия) и суммарный оптический отклик от нулевого, первого и второго дифракционного максимумов (штрих) для $\lambda=405 \mathrm{~nm}$.

причем период осцилляций всех зависимостей одинаков и уменьшается с увеличением угла падения. Это свидетельствует об одинаковой природе этих осцилляций. Относительные амплитуды осцилляций $\delta_{m}(\varphi)$ и $R_{0}(\varphi)$ практически совпадают $(0.22-0.25)$, у $R_{1}(\varphi)$ эта величина составляет примерно 0.35 , а у $R_{\text {sum }}(\varphi)$ она почти на порядок меньше - 0.06. Таким образом, при использовании объектива оптический и магнитооптический отклик от поверхностно неоднородной структуры становится практически таким же, как от однородной поверхности, т.е. теряется информация об особенностях поверхности.

Подобные закономерности наблюдались и при $\lambda=$ $=405 \mathrm{~nm}$. На рис. 8 приведены угловые зависимости коэффициента отражения для нулевого дифракционного максимума $R_{0}(\varphi)$ и $R_{\text {sum }}(\varphi)$, измеренного с использованием апертурной линзы (объектива) при $\lambda=405 \mathrm{~nm}$. Таким образом, наблюдаемые эффекты не зависят от длины волны падающего электромагнитного излучения.

Из рис. 7 видно, что петли гистерезиса, полученные на нулевом дифракционном максимуме, подобны, а при некоторых углах полностью совпадают с петлями, измеренными с использованием объектива. Это свидетельствует о том, что в данном случае для получения объективной информации о магнитных свойствах объемных структур достаточно использовать нулевой дифракционный максимум. Таким образом, применение нулевого дифракционного максимума при магнитооптическом исследовании объемно структурированных систем существенно расширяет угловой диапазон фиксации отраженной волны, а также упрощает процесс измерения.

Проведенные эксперименты на различных структуpax типа фотонных кристаллов и анализ полученных результатов свидетельствуют о том, что в указанном диапазоне размеров элементарных рассеивателей (порядка нескольких микрон), составляющих магнитофотонный кристалл, больших по сравнению с длиной волны падающего электромагнитного излучения, структуры ведут себя как трехмерные объекты. Результирующий магнитооптический отклик (МОЭЭК) системы типа магнитофотонного кристалла включает как коллективные интерференционные явления от упорядоченных элементарных рассеивателей, так и эффекты, связанные с дифракционными особенностями рассеяния света на отдельном элементе структуры, а также наложение эффектов различного уровня трехмерной структуры. Важным моментом рассеяния электромагнитного излучения на указанных структурах является наличие резонансных явлений при выполнении условий, когда имеет место кратность половине длины волны падающего электромагнитного излучения характерных размеров рассеивателей. Эта особенность связана с тем фактом, что основу наблюдаемых эффектов составляют интерференционные и дифракционные явления. Вклад в результирующий отклик вносят индивидуальные характеристики рассеивателей, а также интерференционные явления от различных элементов структур [11], обладающих, вообще говоря, разными магнитными свойствами. Детальный анализ экспериментальных данных позволяет выделить вклады различных компонентов в результирующий отклик. Подобный вывод можно сделать исходя из того факта, что одинаково приготовленные образцы в виде полосок с отжигом и без отжига демонстрируют различное поведение магнитного гистерезиса. В случае с отжигом не наблюдается характерного образования в виде „гребня“ (рис. $4, a, d)$, что свидетельствует об однородности магнитных свойств отожженных образцов, тогда как для неотожженных образцов магнитные свойства слоя кобальта на стенках и на дне структуры отличаются от магнитных свойств пленки на верхних гранях структур. Это отражается в появлении характерного „гребня“ в структуре магнитного гистерезиса при определенных углах падения электромагнитного излучения.

\section{Заключение}

Коротко суммируя результаты представленного исследования, можно сделать вывод, что вклады в наблюдаемые осцилляционные явления различных характеристик для максимумов разных порядков дает интерференция вдоль вертикального направления объемных структур. Эти интерференционные явления в вертикальном на- 
правлении отражают, в частности, свойства верхних и нижних слоев структуры, а также характеристики стенок исследуемых трехмерных объектов.

В настоящей работе исследованы структуры типа магнитофотонного кристалла, вертикальный размер которых много больше длины волны падающего излучения. Исследования показали, что нулевой дифракционный максимум адекватно отражает магнитную составляющую магнитооптического отклика. Кроме того, нулевой дифракционный максимум позволяет извлечь информацию о вертикальном размере структуры. Показано, что при измерении интегрального отклика, полученного с помощью объектива, на угловых зависимостях коэффициента отражения и величины магнитооптического сигнала исчезают интерференционные явления.

Результаты настоящей работы могут быть использованы при анализе сигналов от структур со сложной топологией, что является важным для разработки комплексных систем, содержащих разнородные по структуре и свойствам компоненты. В этой связи следует отметить неизменный интерес к использованию наноструктурированных систем в виде плотных массивов регулярно расположенных вертикальных колонн для создания сверхплотной магнитной памяти [4,19]. Считывание информации с такой структуры связано с эффектами взаимодействия электромагнитного излучения с регулярно структурированной магнитной средой (bit-patterned media). Полученные результаты свидетельствуют об общности исследованных явлений, относящихся к широкому кругу дифракционных и интерференционных проблем, которые находят свое возможное применение в различных технических и научных аспектах.

Авторы выражают свою искреннюю признательность Амирову И.И. за помощь в изготовлении структур. Фотографии структур сделаны на оборудовании ЦКП „Диагностика микро- и наноструктур“ при финансовой поддержке Министерства образования и науки Российской Федерации.

\section{Список литературы}

[1] Еременко В., Жефруа О., Новосад В., Пантье Б., Пишко В., Суш И. // Письма в ЖЭТФ. 1997. Т. 66. Вып. 7. C. $466-469$.

[2] Chetvertukhin A.V., Grunin A.A., Dolgova T.V., Inoue M., Fedyanin A.A. // J. Appl. Phys. 2013. Vol. 113. P. 17A942.

[3] Grimsditch M., Vavassori P. // J. Phys.: Condens. Matter. 2004. Vol. 16. P. R275-R294.

[4] Joel K.W. Yang, Yunjie Chen, Tianli Huang, Huigao Duan, Naganivetha Thiyagarajah, Hui Kim Hui, Siang Huei Leong, Vivian Ng. // Nanotechnology. 2011. Vol. 22. P. 385301.

[5] Costa-Kramer J.L., Alvarez-Sanchez R., Bengoechea A., Torres F., Garcia-Mochales P., Briones F. // Phys. Rev. B. 2005. Vol. 71. 104420.

[6] Vavassori P., Metlushko V., Osgood III R.M., Grimsditch M., Welp U., Crabtree G., Fan Wenjun, Brueck S.R.J., Ilic B., Hesketh P.J. // Phys. Rev. B. 1999. Vol. 59. N 9. P. 6337-6343.
[7] Grimsditch M., Vavassori P., Novosad V., Metlushko V., Shima H., Otani Y., Fukamichi K. // Phys. Rev. B. 2002. Vol. 65. P. 172419.

[8] Guedes I., Grimsditch M., Metlushko V., Vavassori P., Camley R., Ilic B., Neuzil P., Kumar R. // Phys. Rev. B. 2002. Vol. 66. P. 014434.

[9] Guedes I., Zaluzec N.J., Grimsditch M., Metlushko V., Vavassori P., Ilic B., Neuzil P., Kumar R. // Phys. Rev. B. 2000. Vol. 62. N 17. P. 11719-11724.

[10] Рахман Ф. Наноструктуры в электронике и фотонике. М.: Техносфера, 2010. 344 c.

[11] Абрамова С.В., Звездин Н.Ю., Изюмов М.О., Папорков В.А., Проказников А.В. // Нано- и микросистемная техника. 2015. № 9. С. 7-23.

[12] Laermer F., Schilp A. // Patent N 5501893. 26.03.1996.

[13] Laermer F., Schilp A. // Patent N 6531068 B2. 11.03.2003.

[14] Бучин Э.Ю., Ваганова Е.И., Наумов В.В., Папорков В.А., Проказников А.В. // Письма в ЖТФ. 2009. Т. 35. Вып. 13. C. 8-17.

[15] Кричевцов Б.Б., Гастев С.В., Ильюшенков Д.С., Кавеев А.К., Соколов Н.С. // ФТТ. 2009. Т. 51. Вып. 1. C. 109-117.

[16] Кособукин В.А., Кричевщов Б.Б. // ФТТ. 2010. Т. 52. Вып. 4. C. $759-765$.

[17] Борн М., Воль $\oint$ Э. Основы оптики. М.: Наука, 1973. 719 с.

[18] Аббанасьева Д.Е., Звездин Н.Ю., Папорков В.А., Проказников А.В. // Микроэлектроника. 2014. Т. 43. № 3. C. 207-211.

[19] Guangyuan Si, Xiaoxiao Jiang, Jiangtao Lv, Qiongchan Gu, Fengwen Wang // Nanoscale Research Lett. 2014. Vol. 9. P. 299. 